\section{A masking approach to the problem of form perception}

\author{
WILLIAM R. UTTAL \\ University of Michigan, Ann Arbor, Michigan 41804
}

The effects of deletion of certain components of a dotted triangle are studied, using a masking technique. Masking interference is achieved by varying the density of randomly placed dots within which stimulus triangles must be detected. The results of the study indicate that deletion of the dots composing the corners of the triangles decreases performance no more than deletion of an equivalent number of randomly chosen dots. However, the detectability of the line of dots composing the sides is functionally related to the recognition of the orientation of the triangle. The results suggest that the relationship of the parts of such a stimulus figure may be more important than the specific parts themselves.

\section{Modern information-processing theories} of visual form perception have been classified by Neisser (1967) into two broad categories: the template matching models and the feature analytic models. Each of these approaches has certain problems associated with its formulation which have made each theory less than fully adequate to describe the exceedingly complex processes underlying the classification and recognition of forms. It is difficult to understand how template matches might work in a stimulus environment in which rotations, translation, and size of the stimulus object cannot be controlled without an enormous, and therefore, unreasonably large set of templates. Similarly, feature analytic models often ignore the fact that either the extraction of specific features or the transformation of a pattern from one set of dimensions into a similar, though smaller, set really does not solve the problem of recognition; it merely thrusts it to another level of discourse.

For many years data have been available to make it clear that form recognition is a far more complicated process than that usually modeled by the information-processing theories. All too often these theories seemed to be based upon either the technology developed for computers or physiological mechanisms observed in simpler animals. Yet these simplistic mechanisms are not able to simulate the more complex aspects of human form perception. Leeper's studies of fractured figures, for example (Leeper, 1935), long ago demonstrated the effectiveness of set or of verbal cues for the recognition of fractured patterns.

Other studies in both speech and visual form recognition with missing parts, such as the recent paper by Warren (1970) or much of the work on perceptual "filling" or "closure" by the Gestalt psychologists, suggest that it may be that "key" or "critical" features are not of prime importance. Rather, the general pattern of relationships among the constituent parts may constitute the critical information in pattern perception. Components of the stimulus patterns do not seem to carry information in isolation; quite to the contrary, they become significant only in terms of their temporal or spatial relation to the other components of the stimulus configuration.

It is also surprising, as one scans the pattern-recognition literature, to see how infrequently experiments are performed which specifically ask this question: What parts, features, or relational characteristics of the visual pattern are necessary or sufficient for the recognition of a given form? Sutherland (1967), in commenting on some papers presented at a symposium on visual and speech form perception, summarized this point succinctly when he pointed out that much of the recent experimental work concentrated on secondary issues, such as the time constants, or labeling of recognition processes rather than what he considered the critical issues, namely, "how it is that the classification process takes place" and the "question of which features of patterns are used in classification."

In the present paper we directly consider this question of what parts, if any, of a geometric form are critical for the recognition of that form. We use a technique which is based upon a stimulus pattern used previously by Julesz (1967) and by McFarland (1967) -a triangle with missing parts. However, in this case we do not present the parts of the triangle in various temporal orders to explore temporal effects as they did. Instead, dotted triangles are used in which specific omissions of certain dots have been made. The stimulus triangles are degraded by the presence of additional random noise dots presented in a continuous stream in random positions both preceding and following the character. I (Uttal, 1969) have called this form of interfering stimulus "dynamic visual noise" (DVN). Twenty triangles, varying in their completeness and their orientation, were used as stimuli. The S's task in all cases was to determine the direction the apex of the triangle was pointing.

The main advantage of this experimental paradigm is that simple figures can be used and only a simple task is required of the $S$, yet the degree of recognizability of the stimulus triangles can be varied continuously from presentations easily recognized down to instances in which the recognition scores vary little from chance levels. The effect of the presence or absence of specific parts of the stimulus pattern can thus be assayed directly with a great deal of precision.

\section{Subjects}

Four Ss, undergraduate students at the University of Michigan (paid on an hourly basis), participated in this experiment. Each had served in other similar experiments for almost an entire semester and must, therefore, be considered a relatively well-trained $\mathrm{O}$ at the time of the experiment. Nevertheless, as we have found in related experiments, the simplicity of the task was such that no long training periods were necessary.

\section{Procedure}

Stimulus materials consisted of more or less complete dotted triangles presented in one of four orientations. The presentation of these stimuli occurred at the midpoint of a $2-\mathrm{sec}$ burst of dotted visual noise of varying density on the face of a cathode-ray oscilloscope (CRT). This CRT was a special unit with a P-21 ultrashort persistence phosphor, the characteristics of which were specified by the manufacturer to be such that any trace decayed to less than $1 / 10$ th of $1 \%$ of its original brightness in less than 50 microsec. However, it should also be noted that in a recent study (Uttal \& Hieronymus, 1970) it was shown that the persistence of the phosphor for a period as long as $20 \mathrm{msec}$ had little effect on masking with DVN. Nevertheless, the use of the ultrashort persistence CRT, in the case of the present experiment, guarantees that any short-term storage of information is a visual one and not an artifact of the equipment. The noise was displayed over a visual angle of 8 by $8 \mathrm{deg}$. The stimulus triangles were about 5 by 5 deg in visual angle.

The random placement of the dots of the DVN was under the control of random-number generators programmed within the context of the digital computer 


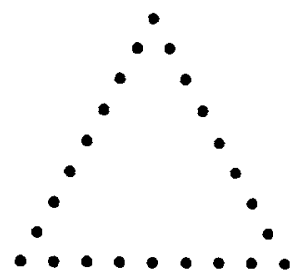

Type I

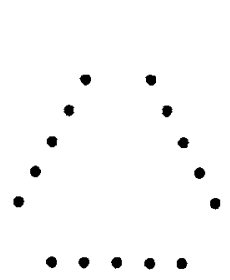

Type II
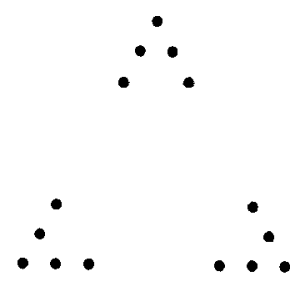

Type II

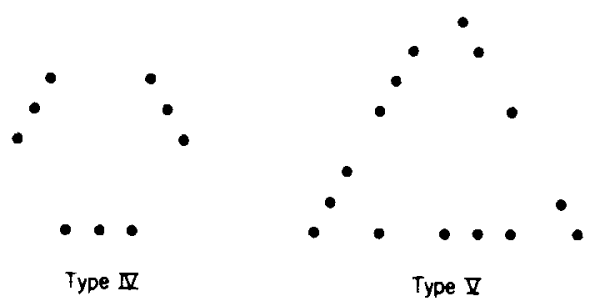

Fig. 1. Diagrams of the five classes of stimulus triangles used in the experiment. All four orientations of the triangle (apex up, down, left, and right) were represented by one each of the five classes, for a total of 20 different stimulus patterns. Each of these stimuli was presented in varying amounts of dynamic visual noise.

control program, which monitored the operation of the entire experinent.

Noise levels (IPI) of $8,6,4,3,2$, and $1 \mathrm{msec}$ were used in descending order on pairs of sequential days; data for two daily sessions were collected for each noise level. Stimulus patterns were gencrated and presented, responses were acquired. and data was analyzed by on-line procedures embodied in the computer program.

The types of stimulus patterns used are shown in Fig. 1. This figure displays the triangles for the upward orientation only. Fifteen other triangles oriented downwards (5), to the left (5), and to the right (5) were also used. A single specinen of the 20 triangles, was randomly selected for presentation in each trial. The stimulus triangles, as can be seen in Fig. 1, consisted of five types in each of the four orientations. Type I was complete witl nine dots on each side. Type II did not contain the three center dots at each of the corners. Type III did not contain the three center dots on each side. and Type IV did not contain any of the five dots at each of the comers. The Type $\mathrm{V}$ configuration dropped random patterns of nine dots from the triangle. For a Type V triangle. the selection of the randomly dropped points was carried out just prior to the presentation of the triangle, but no record was kept of the specitic form of the randomily mutilated stinuli. Eurlier tests of the random-number generator had shown little deviation from randomness.

During each daily session, the same random-number algorithm was used to select the order of presentation of the 20 stimulus triangles. The $S$ s forced-choice responses were chosen from the fout alternatives specifying the direction of orientation of the triangle. The $S$ responded by orally stating, over an intercommunication system to it transcriber who keyed this information into the computer, the perceived orientation of the triangle. This input information was accumulated as the data base and also triggered the presentation of the next trial. At the end of $\% h$, the transcriber and the $O$ changed places, so that each daily session for each of the two Ss lasted for only $1 / 2 \mathrm{~h}$. During that period of time. 200-250 trials were completed. For the eight $S$ sessions for each noise level, therefore, the results of over 1,600 trials were pooled for the plotting of each data point.

\section{RESULTS AND DISCUSSION}

The results of the experiment are shown in Figs. 2 and 3. In Fig. 2 the data have been factored out on the basis of the orientation of the triangle and plotted against the density of the noise level. Clearly, there is no substantial effect of the orientation of the triangle. This result confirms the findings of an earlier experiment (Uttal, Bunnell, \& Corwirs, 1970) on straight-line detection and orientation.

Figure 3 also displays the data as at function of the noise level. but, in this case, the data have been factored on the basis of the five types of displayed triangles. The results of this analysis indicate that the deletion of the three dots at each angle of the triangle caused almost cxactly the same decrement in

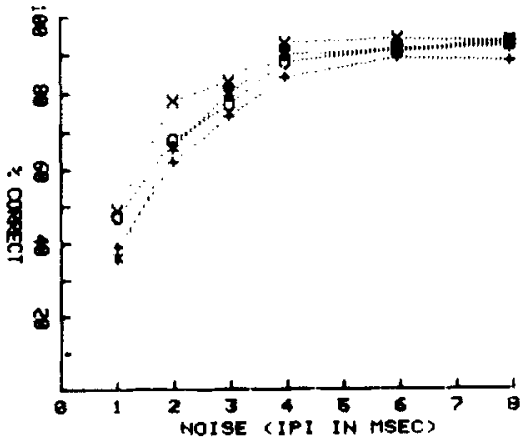

Fig. 2. The data of the experiment factored out according to the orientation of the triangles and plotted as a function of the density of the dynamic visual noise. The clustering of the curves indicates that there is little effect of orientation on the recognition of the triangles. In this figure and in Fig. 3, IPI refers to the interval between the dots of the dynamic visual noise.

recognizability as did the random deletion of nine dots from the figure. On the other hand, the deletion of three dots from the center of the line resulted in a more substantial decrease. Even more damaging to the recognizability of the character was the deletion of all but three of the side points.

The general interpretation which seems to best explain these data is that the sides of the dotted triangle and the relationships among them are the most important features affecting the recognizability of the character. The corners themselves have no more effect in the recognition of the character than does the deletion of an

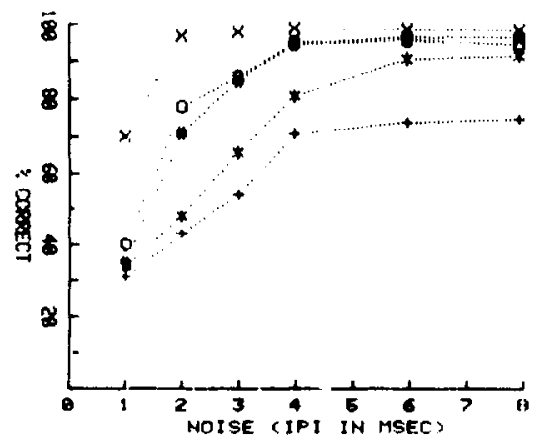

Fig. 3. The data of the experiment factored out according to the type of triangle and plotted as a function of the density of the dynamic visual noise. $X$ plots the data points for the complete triangles (Type $\mathrm{I}$ ); 0 , the data points for the triangle with random deletions (Type V); stimuli; * the data points for Type III stimuli, and + , the data points for the Type IV stimuli. 
equal number of random points. This result flies in the face of the fact that the information content of the dots of the corners is greater than that of the dots of the side. Information theoretical measures would predict a richness of significance, or, more formally, of "information," of the dots of the corner regions. This is because it is at the corners that the redundancy of each part of the pattern is low, due to the discontinuities in the direction of the line, and each corner dot thus conveys more information.

But, while triangles in the context of the present experiment do seem to be characterized more by the sides than by the corners, there are major difficulties in generalizing this notion to other stimulus configurations. It is quite obvious that if the mask itself had had some structure the results might have been quite different. For example, if the noise dots had not been randomly placed but had themselves been organized into dotted lines, the corners might have appeared to be the critical features because of their additional distinctiveness in that environment. On the other hand, if the stimulus triangles had been solid rather than dotted, masking noise composed of randomly placed small angles, similar to the corners, might have replicated the results of this present experiment. The advantage of the present paradigm lies in the fact that the components of the noise and stimulus figures are identical, and, thus, the differences that do emerge are due purely to the organization of the components and are not due to similarities or differences between the components of the stimuli and the noise.

These data make it clear that the recognizability of the triangles is a function of the detectability of the line segments.
The characteristics of line detectability have been discussed in an earlier paper (Uttal, Bunnell, \& Corwin, 1970). In that study it was observed that the spacing of the dots was the critical factor and that lines did not increase in visibility as the number of dots was increased beyond a count of four. The present experiment adds another interesting note to our knowledge of dotted straight-line detection, for it appears that gaps equal in size to the length of the dotted segment itself, typified by the Type III stimuli, still are not sufficiently wide to negate the integrative effect of the two line segments; the Ss are able to detect two three-dot segments separated by a space as large as the three-dot segment better than they are able to detect a three-dot segment by itself. The extent of this sort of spatial integration is yet to be fully described or explained.

In conclusion, preliminary and simple experiments such as the present one are hard pressed to provide a compelling argument for a new theory of form recognition. They do, however, add to the growing body of evidence which suggests that perhaps too much attention has been attached to theories of form perception that are based upon analysis of the figure into its component features. The results of the present experiment suggest further that it is the relationships among the parts which may be more important to form recognition than the nature of the parts themselves. Thus, in a psychological space that familiar three-sided figure is not so much a "tri-angle" as it is a three-sided figure with the three sides in an appropriate geometrical relationship. The angles, which convey large amounts of information about these relationships in the geometrical world, offer little help to the pattern-recognition process, at least in the context of this experiment. It is not clear yet how we are going to be able to quantify such metrics as "organization" or "relationship," but it is clear that perhaps there is more of significance in most kinds of human form recognition than simply the constituent elements, features, or parts.

\section{REFERENCES}

JULESZ, B. Some recent studies in vision relevant to form perception. In W. Wathen-Dunn (Ed.), Models for the perception of speech and visual form. Cambridge, Mass: M.I.T. Press, 1967. Pp. 136-154.

LEEPER, R. A study of a neglected portion of the field of learning: The development of sensory organization. Journal of Genetic Psychology, 1935, 46, 41-75.

McFARLAND, J. Some evidence bearing on operations of "analysis" and "integration" in visual form perceptions by humans. In W. Wathen-Dunn (Ed.), Models for the perception of speech and visual form. Cambridge, Mass: M.I.T. Press, 1967. Pp. 212-219.

NEISSER, U. Cognitive psychology. New York: Appleton-Century-Crofts, 1967.

SUTHERLAND, N. S. Comments on the session. In W. Wathen-Dunn (Ed.), Models for the perception of speech and visual form. Cambridge, Mass: M.I.T. Press, 1967. Pp. 239-243.

UTTAL, W. R. Masking of alphabetic character recognition by dynamic visual noise (DVN). Perception \& Psychophysics, 1969, 6, 121-128.

UTTAL, W. R., BUNNELL, L., \& CORWIN, S. On the detectability of straight lines in visual noise: An extension of French's paradigm into the submillisecond domain. Perception \& Psychophysics, 1970, 8, 385-388.

UTTAL, W. R., \& HIERONYMUS, $R$. Spatio-temporal effects in visual gap detection. Perception \& Psychophysics, 1970, 8, 321-325.

WARKEN, R. M. Perceptual restoration of missing speech sounds. Science, 1970, 167, 392-393.

(Accepted for publication July 29, 1970.) 